\title{
Impact of radiotherapy treatment on Jordanian cancer patients' quality of life and fatigue
}

\author{
kholoud Abu obead ${ }^{1}$, Abdul-Monim Batiha ${ }^{2} *$, Mohammed Said Al-Jauissy ${ }^{1}$, Fadwa Alhalaiqa ${ }^{2}$ \\ Mohammed AlBashtawy \\ ${ }^{1}$ Faculty of nursing, Jordan university of science and technology, Jordan \\ ${ }^{2}$ Faculty of nursing, Philadelphia university, Jordan \\ ${ }^{3}$ Princess Salma faculty of nursing, Al al-Bayt university, Jordan \\ *Corresponding author E-mail: abdtiha@philadelphia.edu.jo
}

\begin{abstract}
Background: The distressing treatment of cancer whether chemotherapy or radiotherapy is associated with fatigue and has negative impact on patient quality of life (QOL).

Objectives: The purposes of this study were to examine the impact of radiotherapy treatment on Jordanian cancer patients' QOL and fatigue, and to explore the relationship between fatigue and QOL.

Methods: One group quasi-experimental correlational design was used with 82 patients who had been diagnosed with cancer and required radiotherapy treatment. QOL was measured using the Functional Assessment of Cancer Therapy-General (FACT-G). Fatigue was measured using Piper Fatigue Scale (PFS). Data were collected over a period of three months, and analyzed using Pearson Product Moment Correlation, descriptive statistics and paired-sample t-test.

Results: Significant differences were found between pre- and post- radiotherapy QOL mean total scores $(\mathrm{t}=19.3$, $\mathrm{df}=79$, $\mathrm{P}<0.05)$, as well as physical, emotional, sexual, and functional wellbeing dimensions. Statistically significant differences were found between pre- and post- radiotherapy fatigue mean total scores $(\mathrm{t}=-8.95, \mathrm{DF}=79, \mathrm{P}<0.05)$, as well as on behavioral, affective, sensory, and cognitive dimensions of PFS. Quality of life total scores correlated significantly and negatively with total fatigue scores $(\mathrm{P}<0.01)$.

Conclusions: Exposure of cancer patient to radiotherapy treatment increased their fatigue level and decreased their QOL. Nurses should assess cancer patients before, during, and after their treatment to design proper interventions to reduce fatigue and enhance QOL.
\end{abstract}

Keywords: Cancer Patients, Fatigue, Piper Fatigue Scale, Quality of Life, Radiotherapy.

\section{Introduction}

(Cancer is considered one of non-communicable diseases (NCD) (WHO 2009, Yoel et al. 2013). It is one of the most important health concerns worldwide (WHO 2009); because the health burden in term of prevalence, cost, mortality and morbidity was increased (WHO 2008, WHO 2009, Jemal et al. 2011). In 2008, around three million cancer cases and 7.6 million cancer deaths were estimated worldwide. More than half of these cases and $64 \%$ of the deaths occurred in developing countries (Jemal et al. 2011). In Jordan, cancer is the second leading cause of death (Jordanian Cancer Registry 2008). In 2008, 4606 (74\%) of Jordanian were diagnosed as new cancer cases, around fifty percent (2274 cases) of those were males and $50.7 \%$ were females (Jordanian Cancer Registry 2008).

Cancer diagnosis is a traumatic event that can have a significant emotional impact on affected patients and their families (Jemal et al. 2011). Patients undergoing treatment for cancer may experience severe symptoms that influenced their psychological, social and physical functioning, disrupt family life and even lead to de-pression, all of which affected quality of life (QOL) (Visser \& Smets 1998, Jemal et al. 2011). Radiotherapy and chemotherapy are most common treatments of cancer (Jemal et al. 2011). These treatments remain devastating agents that alter patients' normal lives (Mackillop et al. 1988). In fact, there is a pervasive impression among many nurses that radiotherapy and chemotherapy often lead to diminished QOL, through affecting all portions of life (Mackillop et al. 1988, Ferrel et al. 1995, Prue et al. 2006). Cancer patients frequently reported fatigue as the most distressing symptom (Haberman 1995, Visser \& Smets 1998, WHO 2009). Cancer related fatigue affected by type of treatment (Ahlberg et al. 2003, Prue et al. 2006). It was noticed that $65-100 \%$ of patients receiving radiotherapy treatment (Haberman 1995) and up to 82-96\% of those receiving chemotherapy suffer from fatigue (Cella \& Tulsky 1990, Whedon et al. 1995, Ahlberg et al. 2005). A prospective study conducted by Janaki et al. (2010) on 90 patients with histologically proven cancer receiving radiotherapy examined the magnitude of fatigue and its implication on the quality of life during radiotherapy. The results revealed that fatigue was primarily present in around $90 \%$ of cases and enlarged gradually over the course of radiotherapy and increased in the last week (Janaki et al. 2010). Fatigue is defined as "a distressing, persistent, subjective sense of physical, emotional, and cognitive tiredness or exhaustion related to cancer or cancer treatment that is not proportional to recent activity and interferes with usual functioning"(National Comprehensive Cancer Network 2010). Additionally, Labourey (2007) defined it as a multidimensional subjective phenomenon with physical (lack of energy), emotional, cognitive, and behavioral aspects (Labourey 2007). The researchers distinguished tiredness from fatigue by stating that tiredness is a normal sensa- 
tion that occurs at certain levels of activity (Glaus et al. 1996, SÖDerberg et al. 2002). In contrast, fatigue is abnormal, excessive tiredness. Fatigue is disproportionate or unrelated to activity and is not re-solved by sleep or rest (Glaus et al. 1996, SÖDerberg et al. 2002). It also negatively affected on patient's well-being (WHO 2008, WHO 2009). Fatigue considered as a main predictors of QOL (Vogelzang et al. 1997, Visser \& Smets 1998). Increased fatigue among cancer patients lead to decrease their QOL (Gupta et al. 2007).

Worldwide, several studies have been conducted to measure QOL and fatigue among cancer patients. However, few of them have focused on the type of treatment (chemotherapy or radiotherapy) and its effect on fatigue and QOL mainly in Jordan and Arab countries. Ahmad \& Alasad (2010) evaluated the QOL of patients with different types of cancer in Jordan and its relationship with nursing care. They found that providing proper nursing care improves cancer patients' QOL (Ahmad et al. 2010). However, they did not focus of cancer- treatment related fatigue. Therefore, the aim of this study is answer the following research questions:

1) Do patients who receive radiotherapy as a primary treatment for their cancer have statistically lower scores of quality of life as measured by FACT-G at the end of their treatment as compared to their scores at the beginning of their treatments?

2) Do patients who receive radiotherapy as a primary treatment for their cancer have statistically higher scores of fatigue as measured by PFS at the end of their treatment as compared to their scores at the beginning of their treatments?

3) What is the relationship between fatigue scores as measured by PFS and QOL scores as measured by FACT-G among Jordanian patients who receive radiotherapy as a primary treatment for their cancer at the end of their treatment?

\section{Methods}

\subsection{Design}

One group quasi-experimental correlational design was used to explore QOL and fatigue among Jordanian patients who receive radiotherapy as a primary treatment for their cancer.

\subsection{Theoretical framework}

The City of Hope-Quality of Life (COH-QOL) conceptual model was used as a framework to guide this study (Grant et al. 1996, Grant et al. 2004)

\subsection{Sample and setting}

A convenience sampling procedure was used to recruit potential participants for this study. To decrease the effect of confounding factors on the results, the patient included if they met the following criteria: (a) 20-60 years old, (b) had no history of psychiatric or mental problem, (c) had radiotherapy for the first time, (d) was treated with radiotherapy only, (e) was able to read, write and understand Arabic, (f) had hemoglobin (Hb) level above 12 $\mathrm{g} / \mathrm{dl}$ at the beginning of the study, (g) had no history of cardiac, respiratory or medical illnesses and (h) was able to give verbal consent to participate in this study.

The sample size was determined by Cohen's (1988) formula (Cohen 1988). Cohen identified three levels for the effect of the sample size when using Pearson Product Moment Correlation: small 0.1, medium 0.3, and large 0.5. Based on this classification and literature review, the medium effect correlation between fatigue and QOL was anticipated for this study. Testing one tailed hypothesis at significant level of alpha 0.05 , the sample size was determined to be 80 participants. Eighty two pa- tients were met the inclusion criteria at Albashier hospital and participated in this study.

\subsection{Instrumentation}

Three instruments were used to collect data in this study:

\subsubsection{Functional assessment of cancer therapy-general (FACT-G)}

The Arabic version of FACT-G was used to elicit data about QOL from Jordanian cancer patients receiving radiotherapy. This instrument was developed to measure four cornerstone dimensions of QOL: physical well-being (6 items); social wellbeing (6 items); emotional wellbeing ( 7 items); and functional wellbeing ( 7 items). Each item is rated on a 5 point scale from 0-4 (Cella 1997). An additional item related to sexual activity was used to measure sexual satisfaction with score ranges between 0-4, with higher score indicates a higher sexual satisfaction. The instrument in its origin was written at a six grade reading level and is available in nine languages including Arabic (Cella 1997). The total scores of the Arabic version of FACT-G range from $0-108$, with higher rating scores reflecting higher QOL. The Arabic version of FACT-G is reported to have high reliability and validity. Content validity index was reported to be 0.95. Cronbach alpha for internal consistency was 0.967 for total scale and from 0.89-0.98 for subscales (Cohen 1988).

\subsubsection{Piper fatigue scale (PFS)}

The Piper Fatigue Scale (PFS) is a multidimensional tool designed to measure the level of fatigue subjectively, and has been widely used in research. It has the potential to differentiate three levels of fatigue; mild, moderate and severe (Piper et al. 1998). PFS com-posed of 22 numerically scaled, " 0 " to " 10 " items that measure four dimensions of fatigue: behavioral (severity) 6 items; affective (meaning) (5 items); sensory (5 items) and cognitive (mood) (6 items). PFS has good validity and reliability (Piper et al. 1998).

\subsubsection{Demographic data Sheet (DDS)}

The Demographic data sheet was developed by the researcher to elicit background information about the patients. It included questions related to age, marital status, and gender, level of education, monthly income, occupation, and care providers at home.

\subsection{Translation of PFS}

After gaining permission from the original author, the PFS instrument was translated to Arabic language to minimize barriers of assessment with participants (Maneesriwongul \& Dixon 2004, Ozlines 2009). The translated version of the instrument was back-translated to ensure content and semantic validity (Ozlines 2009). Content validity was assessed by a panel of expert in nursing who reviewed the items for comprehensiveness, relevance, clarity, understandability, and ease of administration. The panel of experts recommended no modifications. Before embarking on the full study, a pilot test of the Arabic version of PFS was conducted with 10 participants within the target population to ensure that the tool is readable and can be understood by those who will use it. The pilot study indicated that Arabic version of PFS was in general readable, and easily understood. Participants did not request any additional information to be included in the questions. Structured interview for each participant required from 10 to 15 minutes. Reliability coefficient alpha was calculated for total PFS scores and subscales scores. The results showed that the Arabic version of PFS is a reliable instrument, with internal consistency of the entire Arabic version of PFS (al-pha=0.947), and for the four sub- 
scales: behavioral, affective, sensory, and cognitive dimension (alpha $=0.915,0.807,0.952$, and 0.864) respectively.

\subsection{Ethical consideration and recruitment procedure}

Ethical approval was obtained from Institutional Review Board at Jordan University of Science and Technology and AlBashir Hospital administration. Daily visits were made to the setting to check for participants who meet the inclusion criteria. Once a participant was identified, verbal consent was obtained after providing adequate information about the significance and purposes of the study. Patients were assured that participation is voluntary, and participants were told to feel free to withdraw at any time. Participants were assured that their confidentiality and privacy would be maintained. The collected data only would be used for the purpose of the study. The researcher interviewed each participant using the designated questionnaires FACT-G, PFS, and DDS of the study two times, immediately before receiving first cycle of radiotherapy and after the end of treatment.

\subsection{Data analysis}

Descriptive statistics were used to describe the participants characteristics. A paired-sample t-test was used for total scores, and for scores on each subscales of FACT-G and PFS to determine the differences pre-post radiotherapy. To test the correlation between QOL scores as measured by FACT-G and fatigue scores as measured by PFS at the end of treatment, Pearson Product Moment Correlation was used.

Table 1: Socio-demographic characteristic of the sample.

\begin{tabular}{|c|c|c|c|c|c|c|}
\hline Character & Category & Frequency & $\%$ & Mean & SD & Range \\
\hline \multirow[t]{2}{*}{ Sex } & Male & 27 & 33.75 & & & \\
\hline & Female & 53 & 66.25 & & & \\
\hline Age in years & & & & 43.13 & 8.998 & $20-60$ \\
\hline \multirow[t]{3}{*}{ Marital Status } & Married & 64 & 80 & & & \\
\hline & Single & 12 & 15 & & & \\
\hline & Widow & 4 & 5 & & & \\
\hline \multirow[t]{2}{*}{ Level of Education } & $0-11$ years & 44 & 55 & & & Illiterate-18 \\
\hline & $>12$ years & 36 & 45 & & & \\
\hline \multirow[t]{3}{*}{ Occupation } & Unemployed & 48 & 60 & & & \\
\hline & Skilled work & 13 & 16.25 & & & \\
\hline & Unskilled work & 19 & 23.75 & & & \\
\hline \multirow[t]{6}{*}{ Patient Care Provider } & Father/Mother & 9 & 11.25 & & & \\
\hline & Brother/ Sister & 4 & 5 & & & \\
\hline & Son/ Daughter & 6 & 7.5 & & & \\
\hline & Husband/Wife & 56 & 70 & & & \\
\hline & Patient himself & 4 & 5 & & & \\
\hline & Others & 1 & 1.25 & & & \\
\hline \multirow[t]{6}{*}{ Type of Cancer } & Breast & 43 & 53.75 & & & \\
\hline & Bladder & 2 & 2.5 & & & \\
\hline & Larynx & 9 & 11.25 & & & \\
\hline & Lymphoma & 2 & 2.5 & & & \\
\hline & Prostate & 4 & 5 & & & \\
\hline & Others & 20 & 25 & & & \\
\hline
\end{tabular}

Table 2: The FACT-G prior receiving first dose of radiotherapy $(\mathrm{N}=80)$.

\begin{tabular}{|c|c|c|c|c|c|c|c|}
\hline Group & & Physical & Social & Emotional & Sexual & Functional & Total scores \\
\hline \multirow[t]{2}{*}{ All participants } & $\mathrm{M}$ & 26 & 21.5 & 10.5 & 3.1 & 23.63 & 84.63 \\
\hline & SD & 3.48 & 2.52 & 2.86 & 0.85 & 4.1 & 8.88 \\
\hline Highest score & & 24 & 24 & 28 & 4 & 28 & 108 \\
\hline
\end{tabular}


Table 3: The PFS scores prior receiving first dose of radiotherapy $(\mathrm{N}=80)$

\begin{tabular}{|c|c|c|c|c|c|c|}
\hline Group & & Behavioral & Affective & Sensory & cognitive & $\begin{array}{l}\text { Total PFS } \\
\text { scores }\end{array}$ \\
\hline \multirow[t]{2}{*}{ All participants } & $\mathrm{M}$ & 0.99 & 0.74 & 0.69 & 2.06 & 0.79 \\
\hline & SD & 0.45 & 0.28 & 0.26 & 1.83 & 0.37 \\
\hline Highest score & & 10 & 10 & 10 & 10 & 10 \\
\hline
\end{tabular}

\subsection{Post treatment measurements}

At the end of radiotherapy treatment for each participant the total FACT-G scores ranged from 49 to 74 ( $M=62.59$, $\mathrm{SD}=4.43$ ). Al-most all participants scored low on all subscales of FACT-G at the end of radiotherapy treatment (see Table 4) (e.g. physical wellbeing scores ranged from 7 to $26(\mathrm{M}=18.04$, $\mathrm{SD}=3.14)$.

The total participants' scores on PFS at the end of radiotherapy treatment ranged from 0.00 to $7.64(\mathrm{M}=2.12, \mathrm{SD}=2.06)$. All participants scored high on all subscales of the PFS at the end of their radiotherapy treatment with behavioral subscale scores ranged from 0.00 to $9.5(\mathrm{M}=2.31, \mathrm{SD}=2.22)$ (e.g. affective subscale scores ranged from 0.00 to $8.8(\mathrm{M}=2.35, \mathrm{SD}=2.22)$ ) (see Table 5).

\subsection{Research question one}

A significant differences between respondents' total mean scores of QOL pre- and post- radiotherapy as measured by FACT-G questionnaire ( $\mathrm{t}=19.3$, df $=79, \mathrm{P}<0.05$ ). In addition, significant statistical differences were found between pre- and post-treatment scores for physical, emotional, sexual, and functional subscales $(\mathrm{t}=18.1,9.5,7.9,18.6, \mathrm{df}=79, \mathrm{P}<0.05)$ respectively. There was no significant differences between respondents' mean scores on social wellbeing subscales $(\mathrm{t}=1.9$, $\mathrm{df}=79$, $\mathrm{P}<0.05$ ) (see Table 6).

\subsection{Research question two}

The findings showed a significant differences between respondents' total mean scores of fatigue pre- and post- radiotherapy $(\mathrm{t}=-8.95$, df $=79, \mathrm{P}<0.05)$. In addition, significant differences were found between pre- and post-radiotherapy scores for behavioral, affective, sensory, and cognitive dimensions subscales $(\mathrm{t}=-08.8,-8.52,-7.06,-3.99, \mathrm{df}=79, \mathrm{P}<0.05)$ respectively (see Table 7).

Table 4: The FACT-G at the end of radiotherapy treatment $(\mathrm{N}=80)$

\begin{tabular}{|c|c|c|c|c|c|c|c|}
\hline Group & & Physical & Social & Emotional & Sexual & Functional & Total scores \\
\hline \multirow{2}{*}{ All participants } & $\mathrm{M}$ & 18.04 & 20.89 & 7.35 & 2.14 & 13.20 & 62.59 \\
\hline & SD & 3.14 & 1.56 & 2.02 & 0.62 & 2.27 & 4.4 \\
\hline Highest score & & 24 & 24 & 28 & 4 & 28 & 108 \\
\hline
\end{tabular}

Table 5: The PFS at the end of radiotherapy treatment $(\mathrm{N}=80)$

\begin{tabular}{|c|c|c|c|c|c|c|}
\hline Group & & Behavioral & Affective & Sensory & cognitive & Total PFS scores \\
\hline \multirow[t]{2}{*}{ All participants } & $\mathrm{M}$ & 2.31 & 2.35 & 2.06 & 2.36 & 2.12 \\
\hline & SD & 2.22 & 2.22 & 1.83 & 2.02 & 2.06 \\
\hline Highest score & & 10 & 10 & 10 & 10 & 10 \\
\hline
\end{tabular}

Table 6: Results of paired-sample t-test for QOL scores

\begin{tabular}{|c|c|c|c|c|c|c|c|}
\hline FACT-G subscales & Time & $\mathrm{N}$ & $\mathrm{M}$ & SD & $\mathrm{t}$ & $\mathrm{df}$ & Sig \\
\hline \multirow[t]{2}{*}{ Physical } & Pre radiotherapy & 80 & 26 & 3.48 & $18.06^{*}$ & 79 & 0.000 \\
\hline & Post radiotherapy & 80 & 18.04 & 3.14 & & & \\
\hline & Pre radiotherapy & 80 & 21.5 & 2.52 & 1.9 & 79 & 0.056 \\
\hline & Post radiotherapy & 80 & 20.89 & 1.56 & & & \\
\hline \multicolumn{8}{|l|}{ Emotional } \\
\hline & Pre radiotherapy & 80 & 10.5 & 2.86 & & & \\
\hline & Post radiotherapy & 80 & 7.35 & 2.02 & $9.5^{*}$ & 79 & 0.000 \\
\hline \multicolumn{8}{|l|}{ Functional } \\
\hline & Pre radiotherapy & 80 & 23.63 & 4.1 & & & \\
\hline & Post radiotherapy & 80 & 13.2 & 2.27 & $18.6^{*}$ & 79 & 0.000 \\
\hline \multicolumn{8}{|l|}{ Sexual } \\
\hline & Pre radiotherapy & 80 & 3.1 & 0.85 & & & \\
\hline & Post radiotherapy & 80 & 2.14 & 0.62 & $7.9^{*}$ & 79 & 0.000 \\
\hline \multicolumn{8}{|l|}{ Total FACT-G } \\
\hline & Post radiotherapy & 80 & $\begin{array}{l}62.59 \\
62.05\end{array}$ & $\begin{array}{l}0.00 \\
4.4\end{array}$ & $19.3^{*}$ & 79 & 0.000 \\
\hline
\end{tabular}

$* \mathrm{P}<0.05$ 


\begin{tabular}{|c|c|c|c|c|c|c|c|}
\hline PFS subscale & Time & $\mathrm{N}$ & M & SD & $\mathrm{T}$ & df & Sig \\
\hline \multirow[t]{2}{*}{ Behavioral } & Pre radiotherapy & 80 & 0.99 & 0.45 & \multirow[t]{2}{*}{$-8.80^{*}$} & \multirow[t]{2}{*}{79} & \multirow[t]{2}{*}{0.000} \\
\hline & Post radiotherapy & 80 & 2.31 & 2.22 & & & \\
\hline \multirow[t]{2}{*}{ Affective } & Pre radiotherapy & 80 & 0.74 & 0.28 & \multirow[t]{2}{*}{$-8.52 *$} & \multirow[t]{2}{*}{79} & \multirow[t]{2}{*}{0.000} \\
\hline & Post radiotherapy & 80 & 2.35 & 2.22 & & & \\
\hline \multirow[t]{2}{*}{ Sensory } & Pre radiotherapy & 80 & 0.69 & 0.26 & \multirow[t]{2}{*}{$-7.06 *$} & \multirow[t]{2}{*}{79} & \multirow[t]{2}{*}{0.000} \\
\hline & Post radiotherapy & 80 & 2.06 & 1.83 & & & \\
\hline \multirow[t]{2}{*}{ Cognitive } & Pre radiotherapy & 80 & 2.06 & 1.83 & \multirow[t]{2}{*}{$-3.99 *$} & \multirow[t]{2}{*}{79} & \multirow[t]{2}{*}{0.000} \\
\hline & Post radiotherapy & 80 & 2.36 & 2.02 & & & \\
\hline \multirow[t]{2}{*}{ Total PFS } & Pre radiotherapy & 80 & 0.79 & 0.37 & \multirow[t]{2}{*}{$-8.95 *$} & \multirow[t]{2}{*}{79} & \multirow[t]{2}{*}{0.000} \\
\hline & Post radiotherapy & 80 & 2.12 & 2.06 & & & \\
\hline
\end{tabular}

Table 8: Results of Pearson Product Moment Correlation Coefficient between Fatigue Scores and QOL Scores

\begin{tabular}{|c|c|c|c|c|c|c|c|}
\hline PFS subscale & Time & $\mathrm{N}$ & $\mathrm{M}$ & SD & $\mathrm{T}$ & $\mathrm{df}$ & Sig \\
\hline \multirow[t]{2}{*}{ Behavioral } & Pre radiotherapy & 80 & 0.99 & 0.45 & \multirow[t]{2}{*}{$-8.80 *$} & \multirow[t]{2}{*}{79} & \multirow[t]{2}{*}{0.000} \\
\hline & Post radiotherapy & 80 & 2.31 & 2.22 & & & \\
\hline \multirow[t]{2}{*}{ Affective } & Pre radiotherapy & 80 & 0.74 & 0.28 & \multirow[t]{2}{*}{$-8.52 *$} & \multirow[t]{2}{*}{79} & \multirow[t]{2}{*}{0.000} \\
\hline & Post radiotherapy & 80 & 2.35 & 2.22 & & & \\
\hline \multirow[t]{2}{*}{ Sensory } & Pre radiotherapy & 80 & 0.69 & 0.26 & \multirow[t]{2}{*}{$-7.06^{*}$} & \multirow[t]{2}{*}{79} & \multirow[t]{2}{*}{0.000} \\
\hline & Post radiotherapy & 80 & 2.06 & 1.83 & & & \\
\hline \multirow[t]{2}{*}{ Cognitive } & Pre radiotherapy & 80 & 2.06 & 1.83 & \multirow[t]{2}{*}{$-3.99 *$} & \multirow[t]{2}{*}{79} & \multirow[t]{2}{*}{0.000} \\
\hline & Post radiotherapy & 80 & 2.36 & 2.02 & & & \\
\hline \multirow[t]{2}{*}{ Total PFS } & Pre radiotherapy & 80 & 0.79 & 0.37 & \multirow[t]{2}{*}{$-8.95 *$} & \multirow[t]{2}{*}{79} & \multirow[t]{2}{*}{0.000} \\
\hline & Post radiotherapy & 80 & 2.12 & 2.06 & & & \\
\hline
\end{tabular}

\subsection{Research question three}

Significant negative relationship between total fatigue scores, and total QOL scores $(\mathrm{r}=-0.381,-0.313,-0.446,-0.322,-0.29, \mathrm{P}<0.01)$ respectively. This means increased total fatigue score was associated with decreased QOL. Increased total fatigue scores significantly decreased physical dimensions of QOL scores $(\mathrm{r}=-0.637$, $0.583,-0.677,-0.565,-0.585, \mathrm{P}<0.01)$ respectively. However, a significant positive relationship was found between total fatigue scores as well as behavioral, affective, sensory and cognitive dimensions of fatigue, and social dimension of QOL scores $(\mathrm{r}=0.307,0.335,0.301,0.242,0.255)$ respectively. This means increased total fatigue scores significantly increase $\mathrm{d}$ social dimension of QOL (see Table 8).

\subsection{Additional results}

For the qualitative results, the following responses were obtained. The duration of fatigue among all participants ranged from one to six weeks. All participants believed that the radiotherapy factor contributes most to fatigue. All participants believed that the cessation of radiotherapy treatment was the best thing to relieve their fatigue.

\section{Discussion}

The findings of this study revealed significant differences between patients' mean scores of QOL pre- and post- radiotherapy were found on FACT-G total scores. Significant differences between patients' mean scores of fatigue pre- and post- radiotherapy were found on PFS total scores. There was a significant negative relationship between fatigue total scores and QOL total scores.

The current findings relating to physical, emotional, sexual, and functional dimensions of QOL is congruent with several research studies (de Graeff et al. 2000, Guren et al. 2003, Bruheim et al. 2010, National Cancer Institue 2012). Presence of significant dif- ferences in scores of physical, emotional, sexual, and functional dimension of QOL in this study could be explained in light of the impact of radiotherapy on human body. Radiotherapy is aggressive treatment that altered patients' normal lives through physiological changes such as fatigue and gastrointestinal tract disturbances (e.g. nausea and vomiting) (Ahlberg et al. 2005). Low level of emotional wellbeing in cancer patient could be attributed to many reasons (e.g. fear of the new, intimidating radiotherapy experience, side effects of radiotherapy, and fear of not responding to radiotherapy treatment (Bjordal \& Kaasa 1995, de Graeff et al. 2000, Ahlberg et al. 2005).

Radiotherapy is disturbed the daily activities of cancer patients (Bjordal \& Kaasa 1995, Guren et al. 2003, Bruheim et al. 2010, National Cancer Institue 2012). It also led to decrease sexual interest and persistent sexual dysfunction (Jensen et al. 2003). Consequently this affect negatively on sexual and functional wellbeing (Guren et al. 2003, Jensen et al. 2003, National Cancer Institue 2012). This result is consistent with our study findings.

In this study there were no significant differences in social wellbeing scores of QOL pre and post radiotherapy. This could be rationalized to the nature of Arabic values and culture (Ahmed et al. 2006, Alhalaiqa et al. 2012). Arabic families have close relationship and ties this provides a good social support sources in comparison with western culture (Barakat 1993, Tawalbeh et al 2013). The reason for such result is that Arabic culture is shaped by the religion of Islam. The teachings of Islam concerning the social responsibilities are based on kindness and consideration of others (Barakat 1993, Ahmed et al. 2006, Alhalaiqa et al. 2012). Based on this, patients who are newly diagnosed with cancer and undergoing radiotherapy treatment for the first time usually receive excessive social support from others especially from their families (Tawalbeh et al 2013). From the other hand, Guren et al (2003) found significant differences between pre- and post- radiotherapy scores in social wellbeing subscale scores. However, this study conducted in western and the result is limited by nature of the sample since most of them were female $53(66.3 \%)$, and married $64(80 \%)$. 
Ahlberg et al (2003) \& Vogelzang et al (1997) found that the fatigue was increased during radiotherapy treatment. Also they found that other symptoms were significantly correlated to fatigue (e.g. loss of appetite, nausea, vomiting and diarrhea) (Ahlberg et al. 2005). Cancer patients who received radiotherapy as a treatment claimed that the fatigue continued more than six weeks after the course of treatment (Vogelzang et al. 1997). This result was also reported by our study's participants, who considered the stopping of radiotherapy as a best treatment for their fatigue.

This study's findings showed significant differences between patients' mean scores of fatigue pre- and post- radiotherapy which consistent with previous studies (Visser \& Smets 1998, Prue et al. 2006, Janaki et al. 2010). In contrast, Brown et al. (2006) found that radiotherapy use, dose and disease site did not have a significant impact on fatigue levels. The reason for increased fatigue scores at the end of treatment of cancer patient could be explained by the complexity and multidimensional source of fatigue (Smets et al. 1996). Radiotherapy cause cellular death (Aistar 1987) as a consequence several chemicals are released into circulation that might increase basal metabolic rate, which might affect energy levels (Aistar 1987, Jensen et al. 2003, Batiha et al. 2013). From the other hand, Using of radiotherapy was associated with poor cognitive wellbeing (Klein et al. 2002). Taphoorn (1994) claimed that there were no differences between pre- and post- radiotherapy scores in affective and cognitive dimensions of fatigue. However, this result was limited by sample since all of them had brain tumor and were diagnosed with cancer for over a year. Also the instrument used by Taphoorn to measure fatigue was different from the instrument used in current study.

The cancer as a diagnosis and its treatment was associated with high level of fatigue which affected on the patient QOL (Vogelzang et al. 1997, Grant et al. 2004, Batiha 2012). Ahlberg et al (2005) found that the increasing level of fatigue lead to significant decrease of QOL (Ahlberg et al. 2005). This could be explained the findings of this study which indicated that high fatigue scores associated with deterioration in physical, emotional, functional domain of QOL. The finding of this study was congruent with previous research (Armstrong et al. 1993, WHO 2008, Janaki et al. 2010, National Comprehensive Cancer Network 2010). Additionally, these findings were reflected the conceptual framework of which we adopted for this study (Grant et al. 2004).

Current findings indicated that the increase in fatigue scores associated with increase in social wellbeing of QOL scores. The social wellbeing of QOL includes the roles and relationships, which define individual life (Curt et al. 2000). Fatigue affected family roles and relationships with patients (So et al. 2003). Patients with cancer who are experiencing fatigue may be unable to perform self-care, maintain usual functioning, and participate in leisure activities (Curt et al. 2000, So et al. 2003). In Islamic religion and Arabic culture, family members are responsible to provide emotional and social support, and act as care providers for patients' who are unable to perform self-care activities (Ahmed et al. 2006 , Alhalaiqa et al. 2012, Batiha \& AL Bashtawy 2013). The QOL is conceptualized as a multidimensional domain, interrelated and over-lapping. Therefore, the manipulation of any of these domains will affect QOL (Grant et al. 2004). High level of fatigue diminished energy of the patients, consequently leading to elimination of some activities, which affected all domains of QOL (Bower et al. 2000, Curt et al. 2000, Batiha et al. 2013).

\section{Limitations and recommendations}

Cancer patients in this study who received radiotherapy treatment were liable to have a high level of fatigue that negatively affected on their QOL. However, this results is limited by using of convenience sample, the small sample size and setting (Albashier hospital).The inferential statistics performed on these data must therefore be interpreted with extreme caution. These limitations were threatening the generalizability of the findings. Future study should increase sample size and include private and public hospitals in Jordan. In this study we use FACT-G and PFS to collect data but these instruments were developed in western and do not measure the spiritual aspect and adaptation of cancer patients. We need to develop an instrument to measure QOL and fatigue that adapt with Arabic cultures and beliefs and to measure patient's adaptation and spiritual wellbeing. Further studies are needed to test the validity and reliability of Arabic version of PFS and to assess knowledge, and attitude toward fatigue among nurses. More research is needed also to explore the relationship between fatigue and QOL in cancer patients treated with other cancer treatment modalities.

It is anticipated that this study will have the potential to motivate staff to take fatigue and quality of life into consideration while providing care for oncology patients. Many gaps in knowledge about cancer-related fatigue and its impact on QOL remain among nurses, and this study is an attempt to fill some of the gaps among Jordanian nurses through increasing their awareness of this symptom, and to bring more rapid positive changes in the patients fatigue response, and improve their QOL. We recommended that the healthcare providers (HCPs) should incorporate fatigue in routine assessments of patients who are being treated for cancer or being followed after completing treatment. They should also design a proper intervention to improve cancer patient QOL.

\section{Acknowledgement}

The authors acknowledge the library staff of Jordan University of science and technology and Philadelphia University of providing articles for this literature review, and to all patients and staff who participates in this study.

\section{References}

[1] Ahlberg K , Ekman T \& Gaston-Johansson F (2005), The experience of fatigue, other symptoms and global quality of life during radiotherapy for uterine cancer. International Journal of Nursing Studies 42, 377-386.

[2] Ahlberg K , Ekman T , Gaston-Johansson F \& Mock V (2003), Assessment and management of cancer-related fatigue in adults. The Lancet 362, 640-650.

[3] Ahmad MM, Alasad JA \& Nawafleh H (2010), Nursing care and quality of life among Jordanian patients with cancer. Leadership in Health Services 23, 233-243.

[4] Ahmed Q, Memish Z, Allegranzi B, Pittet D \& Challenge W (2006), Muslim health care workers and alcohol based handrubs. Lancet 367 , 1025-1027.

[5] Aistar J (1987), Fatigue in Cancer patients: a conceptual approach to clinical problem. Oncology Nursing Forum 14, 25-30.

[6] Alhalaiqa F, Deane K, Nawafleh A, Clark A \& Gray R (2012), Adherence therapy for medication non-compliant patients with hypertension: a randomised controlled trial. Journal of Human Hypertension 26, 117-126.

[7] Armstrong C, Mollman J, Corn B, Alavi J \& Grossman L (1993), Effects of radiation therapy on adult behavior: Evidence for a rebound phenomenon in a phase 1 trial. Neurology 43, 1961-1965.

[8] Barakat H (1993). The Arab World: Society, Culture and State. Burkely: university of California press.

[9] Batiha A-M \& AL Bashtawy M (2013), Knowledge of Philadelphia University students regarding blood Donation. Transfusion Medicine 23, 195-198.

[10]Batiha A-M (2012), Pain Management Barriers in Jordanian Critical Care Units. International Journal of Advanced Nursing Studies 1(2), 73-83.

[11]Batiha A-M, Bashaireh I, Albashtawy M \& Shennaq S (2013), explores the competency of the Jordanian intensive care nurses towards endotracheal tube and oral care practices for mechanically ventilated patients: an observational study. Global Journal of Health Science 5, 203-213.

[12]Bjordal K \& Kaasa S (1995), Psychological distress in head and neck cancer patients 7-11 years after curative treatment. Br J Cancer 71, 592-597. 
[13]Bower JE, Ganz PA, Desmond KA, Rowland JH, Meyerowitz BE \& Belin TR (2000), Fatigue in Breast Cancer Survivors: Occurrence, Correlates, and Impact on Quality of Life. Journal of Clinical Oncology $18,743$.

[14]Brown P , Clark M , Atherton P , Huschka M , Sloan J , Gamble G Girardi J , Frost M , Piderman K \& Rummans T (2006), Will improvement in quality of life (QOL) impact fatigue in patients receiving radiation therapy for advanced cancer. American Journal of Clinical Oncology 29, 52-58.

[15]Bruheim K , Guren MG , Skovlund E , Hjermstad MJ , Dahl O , Frykholm G , Carlsen E \& Tveit KM (2010), Late Side Effects and Quality of Life After Radiotherapy for Rectal Cancer. International Journal of Radiation Oncology*Biology*Physics 76, 1005-1011.

[16]Cella D (1997), The Functional Assessment of Cancer TherapyAnemia (FACT-An) Scale: a new tool for the assessment of outcomes in cancer anemia and fatigue. Seminars in hematology 34, 13-19.

[17]Cella DF \& Tulsky DS (1990), Measuring quality of life today: methodological aspects. Oncology (Williston Park, N.Y.) 4, 29-38; discussion 69.

[18]Cohen J (1988), Statistical power analysis for the behavioral science Hillsdale, N.J: Lwarence Eribaum.

[19]Curt GA, Breitbart W, Cella D, Groopman JE, Horning SJ, Itri LM, Johnson DH, Miaskowski C, Scherr SL, Portenoy RK \& Vogelzang NJ (2000), Impact of cancer related fatigue on the lives of patients: new findings from the fatigue coaliation. The Oncologist 5, 353-360.

[20]de Graeff A , de Leeuw JRJ , Ros WJG , Hordijk G-J , Blijham GH \& Winnubst JAM (2000), Long-Term Quality of Life of Patients With Head and Neck Cancer. The Laryngoscope 110, 98-106.

[21]Ferrel B, Dow K, Leigh L \& Gulasekarm P (1995), Quality of life in long term cancer survivors. Oncology Nursing Forum 22, 915-922.

[22] Glaus A, Crow R \& Hammond S (1996), a qualitative study to explore the concept of fatigue/tiredness in cancer patients and in healthy individuals. Supportive Care in Cancer 4, 82-96.

[23]Grant M, Dean G, Funk B \& Ferrell B (1996), "Bone tired": the experience of fatigue and impact on QOL. Oncology Nursing Forum 23, 539-1547.

[24]Grant M, Ferrell B, Dean G, Uman G, Chu D \& Krouse R (2004), Revision and psychometric testing of the City of Hope Quality of Life-Ostomy Questionnaire. Quality of Life Research 13, 1445-1457.

[25]Gupta D , Lis CG \& Grutsch JF (2007), The Relationship Between Cancer-Related Fatigue and Patient Satisfaction with Quality of Life in Cancer. Journal of Pain and Symptom Management 34, 40-47.

26]Guren MG , Dueland S , Skovlund E , Fosså SD , Poulsen JP \& Tveit KM (2003), Quality of life during radiotherapy for rectal cancer. European Journal of Cancer 39, 587-594.

[27]Haberman M (1995), the meaning of cancer therapy: Bone marrow transplantation as an exemplar of therapy. Seminars in Oncology Nursing 11, 23-31.

[28]Janaki M , Kadam A, Mukesh S , Nirmala S , Ponni A, Ramesh B \& Rajeev A (2010), Magnitude of fatigue in cancer patients receiving radiotherapy and its short term effect on quality of life. Journal of Cancer Research and Therapeutics 6, 22-26.

[29]Jemal A, Bray F, Center MM, Ferlay J, Ward E \& Forman D (2011), Global cancer statistics. A Cancer Journal for Clinicians 61, 69-90.

[30]Jensen PT, Groenvold M, Klee MC, Thranov I, Petersen MA \& Machin D (2003), Longitudinal study of sexual function and vaginal changes after radiotherapy for cervical cancer. International Journal of Radiation Oncology*Biology*Physics 56, 937-949.

[31]Jordanian Cancer Regisrty (2008), National cancer statistics, Cancer Incidence in

Jordan http://www.khcc.jo/National_cancer_statistics.aspx.

[32]Klein M , Heimans JJ , Aaronson NK, van der Ploeg HM , Grit J , Muller M , Postma TJ , Mooij JJ , Boerman RH , Beute GN , Ossenkoppele GJ , van Imhoff GW , Dekker AW , Jolles J , Slotman BJ , Struikmans H \& Taphoorn MJB (2002), Effect of radiotherapy and other treatment-related factors on mid-term to long-term cognitive sequelae in low-grade gliomas: a comparative study. The Lancet 360 , $1361-1368$

[33]Labourey JL (2007), Physical activity in the management of cancerrelated fatigue induced by oncological treatments. Annales de Réadaptation et de Médecine Physique 50, 450-454.

[34]Mackillop WJ, Stewart WE, Ginsburg AD \& Stewart SS (1988), Cancer patients' perceptions of their disease and its treatment. British journal of cancer $58,355-358$.

[35] Maneesriwongul W \& Dixon J (2004), Instrument translation process: a methods review. Journal of Advanced Nursing 48, 175-186.

[36]National Cancer Institue (2012), Factors Affecting Sexual Function in People
http://www.cancer.gov/cancertopics/pdq/supportivecare/sexuality/Hea lthProfessional/page2.

[37] National Comprehensive Cancer Network (2010), NCCN Clinical Practice Guidelines in Oncology ${ }^{\mathrm{TM}}$ : Cancer-related fatigue. http://www.nccn.org/professionals/physician_gls/ PDF/fatigue.pdf.

[38] Ozlines U (2009), Back translation as a means of giving translators a voice. The International Journal for Translation and Interpreting 1(2), $1-15$.

[39]Piper B , Dibble S, Dodd M , Weiss M, Slaughter R \& Paul S (1998), The revised piper fatigue scale: psychometric evaluation in women with breast cancer. Oncology Nursing Forum 25, 677-684.

[40]Prue G , Rankin J , Allen J , Gracey J \& Cramp F (2006), Cancerrelated fatigue: A critical appraisal. European Journal of Cancer 42, 846-863.

[41]Smets E, Garssen B, Cull A \& de Haes J (1996), Application of the multidimensional fatigue inventory (MFI-20) in cancer patients receiving radiotherapy. British journal of cancer 73, 241-245.

[42] So WKW, Dodgson J \& Tai JWM (2003), Fatigue and quality of life among Chinese patients with hematologic malignancy after bone marrow transplantation. Cancer Nursing 26, 211-219.

[43]SÖDerberg SIV, Lundman B \& Norberg A (2002), The meaning of fatigue and tiredness as narrated by women with fibromyalgia and healthy women. Journal of Clinical Nursing 11, 247-255.

[44]Taphoorn M (1994), Cognitive functions and QOL in-patients with low-grade gliomas: The impact of radiotherapy. Neurology 36, 48-54.

[45] Tawalbeh L, Ahmad Tubaishat A, Batiha A-M, Manar Al-Azzam M \& AlBashtawy M (2013), The Relationship Between Social Support and Adherence to Healthy Lifestyle Among Patients With Coronary Artery Disease in the North of Jordan Clinical Nursing Research. DOI: $10.1177 / 1054773813501194$.

[46] Visser M \& Smets E (1998), Fatigue, depression and quality of life in cancer patients: how are they related? Support Care Cancer 6, 101108.

[47] Vogelzang NJ, Breitbart W, Cella D, Curt GA, Groopman JE, Horning SJ, Itri LM, Johnson DH, Scherr SL \& Portenoy RK (1997), Patient, caregiver, and oncologist perceptions of cancer-related fatigue: results of a tripart assessment survey. The Fatigue Coalition. Seminars in hematology 34, 4-12.

[48]Whedon M , Stearns D \& Mills LE (1995), Quality of life of longterm adult survivors of autologous bone marrow transplantation. Oncology Nursing Forum 22, 1527-1535; discussion 1535-1527.

[49]WHO (2008). World cancer report http://www.iarc.fr/en/publications/pdfs-online/wcr/2008/index.php.

[50]WHO (2009). 2008-2013 Action plan for the global strategy for the prevention and control of noncommunicable diseases, World Health Organisation.

http://whqlibdoc.who.int/publications/2009/9789241597418_eng.pdf. (Accessed 2/11/2013).

[51] Yoel U, Abu- Behind the scenes of adherence in a minority population. Israel Hammed T, Cohen A, Aizenberg A, Vardy D \& Shvartzman P (2013), Medical Association Journal: Imaj 15, 17-22. 